\title{
TLR4 Antagonism Reduces Movement-Induced Nociception and ATF-3 Expression in Experimental Osteoarthritis
}

\author{
Joana Ferreira-Gomes ${ }^{1,2}$ \\ Miguel M Garcia ${ }^{3-5}$ \\ Diana Nascimento ${ }^{1,2}$ \\ Lígia Almeida (iD) ${ }^{1,2}$ \\ Ernesto Quesada ${ }^{3,5}$ \\ José Manuel Castro- \\ Lopes (iD) ${ }^{1,2}$ \\ David Pascual iD ${ }^{3-5}$ \\ Carlos Goicoechea ${ }^{3-5}$ \\ Fani Lourença Neto (iD) 1,2 \\ 'i3S - Instituto de Investigação e Inovação \\ em Saúde, Universidade do Porto, Porto, \\ Portugal; ${ }^{2}$ Departamento de Biomedicina, \\ Faculdade de Medicina da Universidade \\ do Porto, Porto, Portugal; ${ }^{3}$ Area of \\ Pharmacology, Nutrition and \\ Bromatology, Department of Basic \\ Health Sciences, Universidad Rey Juan \\ Carlos, Unidad Asociada I+D+i Instituto \\ de Química Médica (IQM) CSIC-URJC, \\ Madrid, Spain; ${ }^{4}$ High Performance \\ Experimental Pharmacology research \\ group, Universidad Rey Juan Carlos \\ (PHARMAKOM), Alcorcón, Spain; \\ ${ }^{5}$ Grupo de Excelencia Investigadora \\ URJC-Banco de Santander-Grupo multi- \\ disciplinar de investigación y tratamiento \\ del dolor (i+DOL), Alcorcón, Spain
}

Correspondence: Joana Ferreira-Gomes Departamento de Biomedicina - Unidade de Biologia Experimental $5^{\circ}$ piso,

Faculdade de Medicina da Universidade do Porto, Rua Dr. Plácido da Costa,

Porto, 4200-450, Portugal

$\mathrm{Tel}+351220426740$

Email jogomes@med.up.pt
Introduction: Toll-like receptor 4 (TLR4) is a pattern recognition receptor involved in the detection of pathogen-associated molecular patterns (PAMPs), but also a "danger-sensing" receptor that recognizes host-derived endogenous molecules called damage-associated molecular patterns (DAMPs). The involvement of TLR4 in rheumatic diseases is becoming evident, as well as its potential role as a target for therapeutic intervention. Moreover, increasing evidence also suggests that TLR4 is implicated in chronic pain states. Thus, in this study, we evaluated whether a systemic administration of a synthetic antagonist of TLR4 (TLR4-A1) could decrease nociception and cartilage degradation in experimental osteoarthritis (OA). Furthermore, as the activation transcription factor (ATF)-3 serves as a negative regulator for TLR4-stimulated inflammatory response, we also evaluated the effect of TLR4 inhibition on ATF-3 expression in primary afferent neurons at the dorsal root ganglia (DRG). Methods: OA was induced in adult male Wistar rats through an intra-articular injection of $2 \mathrm{mg}$ of sodium mono-iodoacetate (MIA) into the left knee. From days 14 to 28 after OA induction, animals received an intraperitoneal injection of either TLR4-A1 $(10 \mathrm{mg} / \mathrm{kg})$ or vehicle. Movement- and loading-induced nociception was evaluated in all animals, by the Knee-Bend and CatWalk tests, before and at several time-points after TLR4-A1/vehicle administration. Immunofluorescence for TLR4 and ATF-3 was performed in L3-L5 DRG. Knee joints were processed for histopathological evaluation.

Results: Administration of TLR4-A1 markedly reduced movement-induced nociception in OA animals, particularly in the Knee-Bend test. Moreover, the increase of ATF-3 expression observed in DRG of OA animals was significantly reduced by TLR4-A1. However, no effect was observed in cartilage loss nor in the neuronal cytoplasmic expression of TLR4 upon antagonist administration.

Conclusion: The TLR4 antagonist administration possibly interrupts the TLR4 signalling cascade, thus decreasing the neurotoxic environment at the joint, which leads to a reduction in ATF-3 expression and in nociception associated with experimental OA.

Keywords: osteoarthritis, TLR4, Knee-Bend, CatWalk, ATF-3, DRG

\section{Introduction}

Osteoarthritis (OA) is a chronic, usually progressive, disease that involves the whole joint in a degenerative process. Pain is the most common manifestation of $\mathrm{OA}$, usually of mechanical origin or activity-related, even though it may become more persistent and also occur at rest and at night in more advanced OA. ${ }^{1}$

Although several factors seem to play a role in the pathogenesis of OA, its underlying mechanisms are still unclear. Current treatments are focused on 
alleviating the symptoms through drugs, such as acetaminophen, Non-Steroidal Anti-Inflammatory Drugs (NSAIDs) and corticosteroids, which do not change the course of the disease and are associated with significant adverse effects, including gastrointestinal bleeding, cardiovascular events and renal toxicity. ${ }^{2-4}$ Therefore, the identification of specific therapeutic targets that allow the development of Disease-Modifying Drugs (DMOADs) is of utmost importance.

Toll-like receptors (TLRs), a family of pattern recognition receptors (PRRs) that recognize structures called pathogen-associated molecular patterns (PAMPs), have been implicated with the development of many pathological conditions. ${ }^{5}$ Recently, a growing body of evidence has suggested that TLR4, a member of the TLRs family, is particularly involved in the pathogenesis of OA. ${ }^{6-8}$ TLR4 expression was shown to be increased in articular cartilage lesions of OA patients, and activation of TLR4 in human chondrocyte cultures induced a variety of inflammatory and catabolic responses that might have a pivotal role in promoting or exacerbating the degradation of cartilage in these patients, including increased expression of IL-1 $\beta$, matrix metalloproteinases (MMP) and nitric oxide (NO), increased prostaglandin E2 (PGE2) synthesis, and decreased aggrecan and type II collagen synthesis., ${ }^{9,10}$ TLR4 increased expression has also been observed in synoviocytes of OA synovium. ${ }^{11}$ On the other hand, several Damage-Associated Molecular Patterns (DAMPs), generated by the injured tissues in OA, can activate TLR4. These include small degradation fragments of hyaluronic acid, fibronectin fragments, the calcium-binding proteins S100-A8 and S100-A9 and the high mobility group protein B1 (HMGB1). After binding to the TLR4 receptor, these molecules can promote cellular innate immune responses, such as the secretion of TNF- $\alpha$, IL- $1 \beta$ and IL-6, which may further propagate this damaging process. ${ }^{12-16}$ Furthermore, oxidative stress and increase of reactive oxygen species (ROS) production have been implicated as a mediator of OA, regulating intracellular signaling processes, chondrocyte apoptosis and senescence, extracellular matrix synthesis and degradation, dysfunction of the subchondral bone and synovial inflammation. ${ }^{17}$ TLR4 can stimulate ROS production via NADPH oxidases, ${ }^{18}$ which are expressed by the chondrocytes and are the main enzymes responsible for ROS formation in synovial fluid, contributing to the progressive cartilage degradation that accompanies OA. ${ }^{17}$
Increasing evidence also suggests a role for TLR4 in the induction and maintenance of chronic pain states. ${ }^{19}$ Tang and colleagues ${ }^{20}$ showed that TLR4 is critical for pain induction after nerve injury in mice, and that spinal targeting of TLR4 with specific antisense oligonucleotides attenuated behavioral hypersensitivity in these animals. Intrathecal siRNA-mediated suppression of TLR4 also attenuated mechanical allodynia and thermal hyperalgesia in a rat chronic constriction injury (CCI) model $^{21}$ and reduced mechanical allodynia and spontaneous pain in a bone cancer pain model. ${ }^{22}$ Therefore, TLR4 seems to be involved not only in OA pathogenesis but also in pain pathophysiology. In patients with OA, chronic pain is ultimately dependent on the activation of primary sensory neurons that innervate the joint. ${ }^{23}$ Since articular cartilage is described as being essentially not innervated, OArelated pain might originate from other affected joint tissues such as the subchondral bone, the synovium and the infrapatellar fat pad. ${ }^{24-26}$ Interestingly, TLR4 expression has been found across glial cells but also in primary afferent neurons of trigeminal and dorsal root ganglia (DRG). ${ }^{27}$ Taking this into account, we hypothesized that targeting TLR4 could offer an effective approach to attenuate pain in OA.

Thus, in this study, we evaluated whether a TLR4 antagonist (TLR4-A1) could efficiently reduce the nociception and cartilage degradation progression in the monoiodoacetate (MIA) rat model of OA. The selected TLR4 antagonist (CAS RN: 1202208-36-3) for this study is a synthetic benzylammonium ether lipid recently described by Peri and colleagues. ${ }^{28}$

Furthermore, as the activation transcription factor (ATF)-3 is induced by TLR4 activation and serves as a negative regulator for TLR4-stimulated inflammatory response in immune cells, ${ }^{29,30}$ and in previous work we observed an increase in ATF-3 expression in primary sensory neurons of OA animals, ${ }^{31}$ we also investigated the effect of TLR4-A1 on ATF-3 expression in DRG neurons.

\section{Materials and Methods}

\section{TLR4-AI Compound}

TLR4-A1 (CAS RN: 1202208-36-3) is a synthetic benzylammonium ether lipid designed like a non-hydrolysable structural analogue of LipoPolySaccharide (LPS), the naturally occurring ligand of TLR4 (Figure 1). Based on recent studies (both in vitro ${ }^{32,33}$ and in vivo ${ }^{28}$ ), this compound proved to be an effective inhibitor of the TLR4 activation pathway. The 


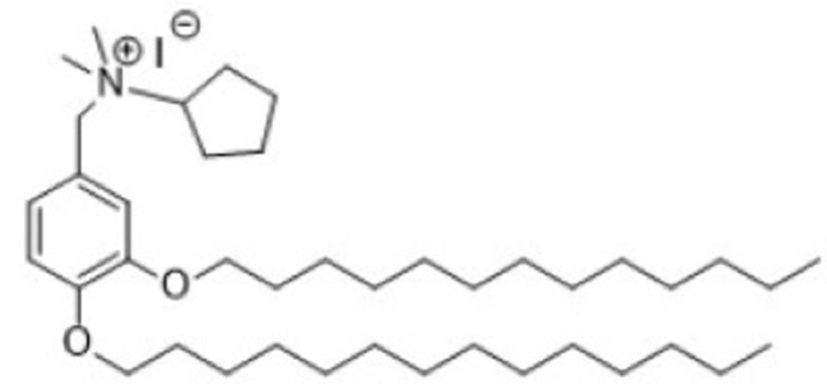

Figure I Structure of the synthetic benzylammonium TLR4 antagonist I (N-(3,4-bis-tetradecyloxy-benzyl)-N-cyclopentyl- $\mathrm{N}, \mathrm{N}$-dimethylammonium iodide; CAS number: 1202208-36-3).

authors reported that TLR4-A1 inhibited LPS-induced TLR4 activation in HEK293 stably transfected with TLR4, MD-2 and CD14 gene. ${ }^{32}$ Furthermore, it was active in blocking TLR4-mediated cytokine production in innate immunity cells. ${ }^{34}$ This molecule was synthesized on large scale following the original procedure described. ${ }^{28}$ The structural identity was verified by NMR studies whereas purity was determined by HPLC analysis prior to administration.

TLR4-A1 has been suggested to interact with TLR4 and its co-receptor protein CD14, making improbable an unspecific selectivity on other TLR, since these lack TLR4/CD14-mediated interaction. ${ }^{32}$

\section{Animals}

Adult male Wistar rats ( $\mathrm{n}=20$; Charles River, France), weighing $230 \pm 30 \mathrm{~g}$ at the beginning of the experiments, were used in this study. Animals were housed in solid bottom cages, with water and food ad libitum, and the animal room was kept at a constant temperature of $22^{\circ} \mathrm{C}$ and controlled lighting ( $12 \mathrm{~h} \mathrm{light} / 12 \mathrm{~h}$ dark cycle). All experimental procedures were performed in accordance with the ethical guidelines for the study of experimental pain in conscious animals, ${ }^{35}$ as well as the European Communities Council Directive 86/609/EEC, and were approved by the Local Committee of Animal Welfare (ORBEA), with all adequate measures being taken to minimize pain or discomfort of the animals.

\section{Induction of Osteoarthritis}

Under brief isoflurane anesthesia, animals were injected intra-articularly, with $25 \mu \mathrm{L}$ of either saline (control group, $\mathrm{n}=8)$ or $2 \mathrm{mg}$ of MIA dissolved in saline $(\mathrm{n}=12$; SigmaAldrich, St Louis, MO, USA). The Hamilton syringe with a $26 \mathrm{G}$ needle was inserted through the patellar ligament into the intra-articular joint space of the left knee. Animals were randomly assigned to each group before the first injection.

\section{Experimental Design}

At the beginning of the experiments, baseline values of movement-induced nociception were evaluated in all animals (day 0) by the Knee-Bend and CatWalk tests, as previously described. ${ }^{36}$ After MIA or saline injection, animals were assessed at day 3,7 and 14, to evaluate if a correct model induction was achieved. At day 14, after the behavioral assessment, each group of animals was randomly divided into two subgroups, each one receiving a daily administration of either vehicle $(10 \%$ ethanol diluted in saline; OA-Vehicle and Control-Vehicle) or of TLR4 antagonist (TLR4-A1, $10 \mathrm{mg} / \mathrm{kg}$ ) dissolved in vehicle (in a 9:1 ratio of $0.9 \%$ saline and ethanol; OA-TLR4A1 and Control-TLR4A1). Therefore, after day 14 the experiments comprised 4 subgroups of rats (OA-Vehicle, Control-Vehicle, OA-TLR4A1 and Control-TLR4A1). Administration of TLR4-A1 and vehicle was performed through an intraperitoneal injection. The dosing regimen chosen was based on Piazza et $\mathrm{al}^{28}$ which showed an increased survival rate from $0 \%$ to $67 \%$, with this compound, in a sepsis mice model.

The effect of the chronic administration of the TLR4A1 or vehicle on the movement-induced nociception of these animals was assessed on days 15, 18, 20, 22, 24, 26 and 28 after saline or MIA injection.

\section{Nociceptive Behavior Evaluation}

Movement-induced nociception was evaluated in all animals by the Knee-Bend and CatWalk tests at days 0, 3, 7, $14,15,18,20,22,24,26$ and 28 post saline or MIA injection. Testing was blind and always done by the same experimenter in order to minimize variability in the application of the tests.

Briefly, the Knee-Bend test consisted on recording the number of squeaks and/or struggle reactions of the animal in response to five flexions and five extensions of the knee joint, according to the following evaluation scale: 0 - no responses to any kind of extension or flexion of the joint; 0.5 - struggle to full range flexion/extension; 1 - struggle to medium range flexion/extension or vocalizations to full range flexion/extension; 2 - squeak reactions in response to medium range manipulations (flexions or extensions) of the joint. The sum of the animal reactions, with a maximum 
of 20, represents the Knee-Bend score, an indication of the animal's nociception induced by movement of the affected joint. For the CatWalk test, animals were placed in a glass platform illuminated such as to reflect the light downwards only at the points of contact of the paw with the glass surface, resulting in a bright sharp image of the paw print. The intensity of the paw print signal increases with the area of the paw in contact with the platform and with the pressure applied by it. The platform was monitored by a video camera with a wide-angle objective, placed under the glass platform and connected to a computer equipped with video acquisition software (Ulead Video Studio, USA). For each hind paw, the total paw print intensity (mean pixel intensity $\mathrm{x}$ number of pixels) was determined, allowing the comparison of the area/pressure applied by each paw. Results were expressed as the percentage of the total ipsilateral paw print intensity (\%TIPPI) in the sum of both paw prints. The CatWalk was always performed prior to the Knee-Bend test to minimize the effect of manipulating the affected knee on the animals' gait.

\section{Tissue Processing}

On day 28 after saline or MIA injection, animals were anaesthetized and perfused with 4\% paraformaldehyde. The DRG from lumbar segments 3, 4 and 5 (L3, L4 and L5) of OA animals were dissected, post-fixed for 4 hours in the same fixative and kept in $30 \%$ sucrose with $0.01 \%$ sodium azide. DRG were serially sliced in $12 \mu \mathrm{m}$ sections using a cryostat (Leica, Germany), and every 10th section was collected in the same glass slide (8-10 sections from each DRG, on average). DRG were oriented to ensure that longitudinal sections were made, and the number of sections obtained from each DRG was similar between animals, giving an indirect measure of this consistency.

The injected knees were also dissected, post-fixed for $72 \mathrm{~h}$ and then decalcified for 8 hours in a buffer containing $7 \% \mathrm{AlCl} 3,5 \% \mathrm{HCOOH}$ and $8.5 \% \mathrm{HCl}$. Afterwards, joints were washed in $0.1 \mathrm{M}$ phosphate buffer saline (PBS) $\mathrm{pH}$ 7.2 and kept in $30 \%$ sucrose with $0.01 \%$ sodium azide until they were cut, in $10 \mu \mathrm{m}$ sections, using a cryostat.

The cutting process was always performed by the same person and with the same method to ensure the consistency of the procedure throughout the study.

\section{Histopathology}

Knee joint sections were stained by the Fast Green and Safranin-O method to evaluate the extent of the histopathological lesions, as previously performed. ${ }^{36}$ The experimenter was blind to the groups. Slides were mounted with Eukitt (Kindler GmbH \& Co, Germany) and images were acquired with an Axioskop-40 microscope equipped with an AxioCam-MRc5 camera (Carl Zeiss MicroImaging GmbH, Germany).

\section{Immunohistochemical Analysis}

Slides containing every tenth section of L3, L4, and L5 DRG of OA animals were used for the immunofluorescence reactions for ATF-3 and TLR4. The immunohistochemistry reactions for each marker were performed in adjacent sections.

Briefly, DRG sections were rinsed in $0.1 \mathrm{M} \mathrm{PBS} \mathrm{pH}$ 7.4, followed by $\mathrm{PBS}+0.3 \%$ triton-X (PBST), and incubated in $10 \%$ normal serum in PBST for $90 \mathrm{~min}$. Sections were then incubated overnight at room temperature with one of the following antibodies diluted in $2 \%$ normal serum in PBST: rabbit anti-ATF-3 (1:500, Santa Cruz Biotechnology Inc, USA); rabbit anti-TLR4 (1:750, Abcam, UK). After thorough PBST washing, sections were incubated with Alexa-Fluor 568 donkey anti-rabbit secondary antibody (1:1000, Molecular Probes, USA) for $1 \mathrm{~h}$ at room temperature. Slides were then rinsed in PBST followed by PBS, mounted with Prolong Gold Antifade medium (Molecular Probes, USA) and coverslipped. Microscopic images of the joints were acquired through an AxioCam MRm with AxioVision 4.6 software (Carl Zeiss MicroImaging GmbH, Germany).

ATF-3 and TLR4 immunolabelled cells were quantified by counting all labelled neurons on a slide containing every tenth section of a DRG (one slide for each reaction, per animal). TLR4 immunolabelling was evaluated separately in the cytoplasm/membrane and in the nuclei. Results were expressed as the number of labelled neurons per section.

\section{Statistical Analysis}

The normality of all data was assessed by the KolmogorovSmirnov test. Behavioral data for movement induced-nociception in control and OA groups were analyzed by two-way repeated measures analysis of variance (RM-ANOVA), for factors time and group, followed by the Tukey's post-hoc test for multiple comparisons between time points within each group. The Unpaired $t$-test was used for the analysis of the immunofluorescence studies. In all statistical analysis, a level of significance of $\mathrm{P}<0.05$ was assumed. 


\section{Results}

\section{Intraperitoneal Injection of TLR4-AI Attenuates Movement-Induced Nociception in OA Animals}

To establish the OA model, MIA was injected into the left knee joint, and movement-induced nociception was evaluated by the Knee-Bend and CatWalk tests. As expected, all OA animals showed a significant increase in the KneeBend score and a significant decrease in the \%TIPPI on the CatWalk test at day 3, when compared with day 0 (before
MIA injection), thereby demonstrating movement-induced nociception as a consequence of OA induction (Figure 2). This movement-induced nociception was also observed on days 7 and 14 post-MIA injection.

On day 14 , OA animals were randomly divided into two groups and treated with vehicle or TLR4-A1 for 14 days. OA rats that received a daily i.p. injection of TLR4A1 showed a reduction in the Knee-Bend score already at day 15, although this decrease in movement-induced nociception was only statistically significant from day 18 onwards, approaching the values of control animals in the

A

\section{Knee-Bend}
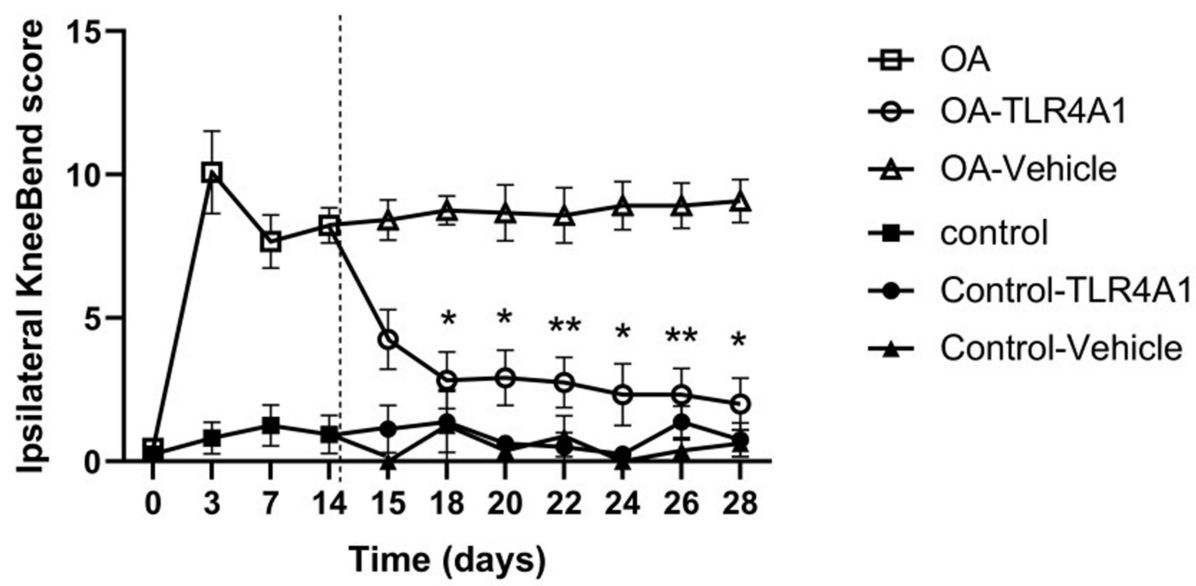

B

\section{Catwalk}

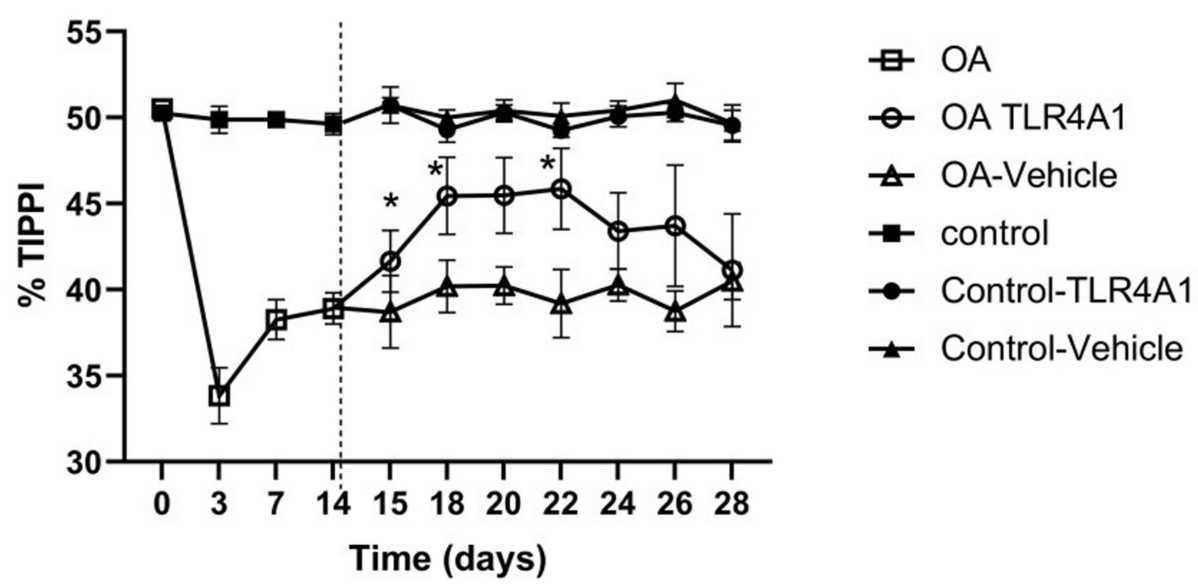

Figure 2 Nociceptive behavior. Nociception associated with movement and loading on the joint was evaluated by the Knee-Bend (A) and CatWalk (B) tests in salineinjected control $(n=8)$ and MIA-injected OA animals $(n=12)$ at baseline (T0), 3, 7 and I4 days. After the behavioral assessment of day I4, each group of animals was randomly divided in two subgroups, Control-Vehicle and Control-TLR4AI ( $n=4 /$ subgroup), OA-Vehicle and OA-TLR4AI ( $n=6 /$ subgroup), and movement-induced nociception was assessed on days 15, 18,20, 22, 24, 26 and 28. Knee-Bend score (A) is presented for the ipsilateral knee. CatWalk data (B) is expressed as the percentage of total ipsilateral paw print intensity (\%TIPPI). Mean \pm SEM, two-way RM-ANOVA followed by Tukey's post-hoc test for multiple comparisons between time points within each group. *P < 0.05 , **P $<0.01$. 
end of the second week (Figure 2A; $\mathrm{P}<0.05$ or $\mathrm{P}<0.01$ ). Regarding the CatWalk test, an increase in \%TIPPI was observed from the beginning of TLR4-A1 administration until day 22 (Figure 2B; $\mathrm{P}<0.05$ ). However, during the second week of treatment, the values of \%TIPPI started to decrease.

OA animals receiving a daily injection of vehicle did not show any difference in the Knee-Bend score and \% TIPPI at any time-points evaluated, maintaining the movement-induced nociception observed prior to intervention (Figure 2).

In both tests, saline-injected control animals showed behavioral responses similar to those observed prior to the injection, during the whole experimental period.

\section{TLR4-AI Intraperitoneal Injection Did Not Improve Cartilage Loss}

Histopathology of the knee joints was performed to evaluate model induction and to observe if TLR4-A1 improved OA histopathological changes. Briefly, no joint damage was observed in both subgroups of saline-injected control animals (Figure 3A and B). In contrast, the MIA-induced animals treated with vehicle showed histopathological alterations similar to what has been described for this $\mathrm{OA} \mathrm{model}^{31}$ (Figure 3C). There was loss of proteoglycan staining and chondrocyte death, accompanied by a marked decrease of the thickness of the articular cartilage with areas of marked erosion and fissures, and exposure of the subchondral bone. OA animals treated with TLR4-A1 did not show any improvement of the histopathological alterations, overlapping with the OA-vehicle subgroup (Figure 3D).

\section{Systemic administration of TLR4}

antagonist induced an increase in nuclear TLR4 expression and had no effect on membrane and cytoplasmic expression

To evaluate if TLR4 levels in DRG neurons were downregulated after TLR4-A1 systemic administration, an immunohistochemistry reaction against TLR4 was performed in OA animals (Figure 4A and B).

Cytoplasmic expression of TLR4 was observed in primary sensory neurons of L3, L4 and L5 DRGs both in OA-vehicle and OA-TLR4A1 subgroups (Figure 4C and D). Although there was as an apparent increase in TLR4 expression in animals receiving the TLR4 antagonist (from $251.7 \pm 33.4$ in the OA-vehicle group to $324.7 \pm 41.0$ in
OA-TLR4A1 animals), it did not reach statistical significance.

Interestingly, an increase in nuclear TLR4 expression was observed upon TLR4-A1 administration (Figure 5A). This increase was observed at the three levels studied although it was more prominent at $\mathrm{L} 3(6.5 \pm 4.3$ in the OA-vehicle group and $22.8 \pm 4.6$ in OA-TLR4A1 animals in $\mathrm{L} 3+\mathrm{L} 4+\mathrm{L} 5$; $\mathrm{P}<0.05$, Figure $5 \mathrm{~B}$ and $\mathrm{C}$ ).

\section{TLR4 Antagonist Suppresses the Expression of ATF-3 in Primary Sensory Neurons}

As ATF-3 serves as a negative regulator for TLR4-stimulated inflammatory response, at least in immune cells, ATF-3 expression was evaluated in primary sensory neurons of MIA-induced animals (Figure 6A and B). The administration of TLR4-A1 significantly decreased the ATF-3 expression induced by OA $(5.7 \pm 0.7$ in the OAvehicle group and $2.4 \pm 0.4$ in OA-TLR4A1 animals, $\mathrm{P}<$ 0.01 ; Figure $6 \mathrm{D}$ ). This reduction was observed at the three lumbar DRGs studied, although it only reached statistical significance at the L4 and L5 levels $(\mathrm{P}<0.05$; Figure $6 \mathrm{C})$.

\section{Discussion}

In this study, we evaluated whether the systemic administration of an antagonist of TLR4 could be effective on decreasing nociception and cartilage degradation in OA, and also neuronal injury. Administration of TLR4-A1 markedly reduced movement-induced nociception on OA animals, particularly in the Knee-Bend test. Moreover, we observed that TLR4-A1 induced a reduction of ATF-3 expression in DRG neurons, but it had no effect on the neuronal cytoplasmic expression of TLR4 nor it improved the histopathology of the joint in our OA animals.

Pain associated with $\mathrm{OA}$ is heterogeneous and involves both peripheral and central neural mechanisms. Evidence suggests that different types of pain with distinct neurobiological mechanisms are part of the OA phenotype. Pain in $\mathrm{OA}$ may be primarily nociceptive, with a protective function, alerting the individual to mechanical limitations of the joints, and to a reduced ability of the joint to cope with additional stress. ${ }^{37}$ In a subset of patients, inflammation plays a pivotal role in the development of $\mathrm{OA}$ and is also a contributor to chronic pain. Primary afferent nerve fibers innervating the joint become exposed to the intraarticular inflammatory milieu, whose components are thought to be the major contributors for the peripheral 

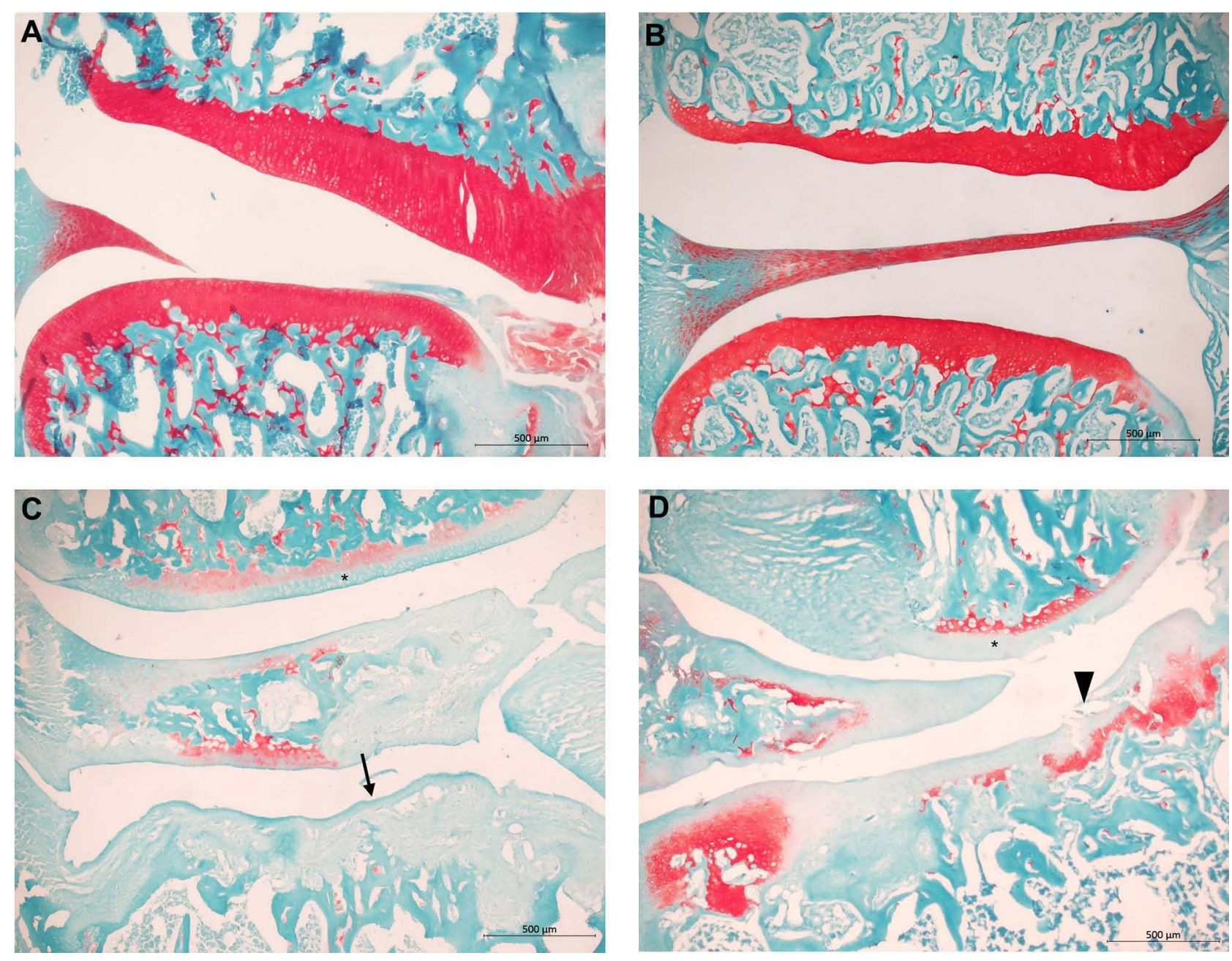

Figure 3 Histopathology of knee joint sections stained with Safranin-O and Fast Green. Control-Vehicle (A) and Control-TLR4AI (B) animals show unaltered articular cartilage (in red) and subchondral bone. OA-Vehicle (C) and OA-TLR4AI animals (D) showed decrease in proteoglycan staining (*), chondrocyte death, loss of intercellular matrix, decrease of the thickness of the articular cartilage (arrow) and erosion of the hyaline articular cartilage (arrowhead).

sensitization of articular mechanonociceptors. ${ }^{38}$ In addition, neuropathic pain mechanisms may also occur during the development of OA. In fact, a recent systematic review showed that the overall prevalence estimate of neuropathic pain in persons with knee or hip OA was $23 \%$, although with considerable heterogeneity. ${ }^{39}$ In a previous study using the MIA-induced model of OA, we have shown two waves of expression of ATF-3, a marker of neuronal injury, in DRG neurons. ${ }^{31}$ The first wave was as early as day 3 post-MIA injection, corresponding to the initial inflammatory stage, which led to the hypothesis that the neuroinflammatory environment of the joint might be neurotoxic for the primary afferents innervating the area. In fact, it is likely that the presence of the pro-inflammatory cytokines IL- $1 \beta$ and TNF- $\alpha$ observed in early OA contribute to that, since it has been shown that they are both able to trigger ATF- $3 .^{40}$ Curiously, besides the known role of
TLR4 in inflammatory conditions, there is also evidence that TLR4 participates in nerve injury. ${ }^{19}$ In this context, TLR4 thus emerges as a possible link between the joint inflammatory environment and the peripheral nerve injury observed in OA, possibly being a promising target for the development of new therapies. Remarkably, the two weeks of i.p. administration of TLR4-A1 significantly reduced movement-induced nociception in OA animals. A more pronounced nociceptive effect in the Knee-Bend test, comparing to the CatWalk, was observed. The Knee-Bend test provides a direct nociceptive stimulation through direct manipulation of the injured joint, while the CatWalk relies on the animal kinematics and load bearing, depending on all the musculoskeletal system of the animal, with the involvement of several neuronal pathways, some of them of supraspinal origin. ${ }^{41}$ This, together with non-improvement of the histopathology alterations of the joint with 


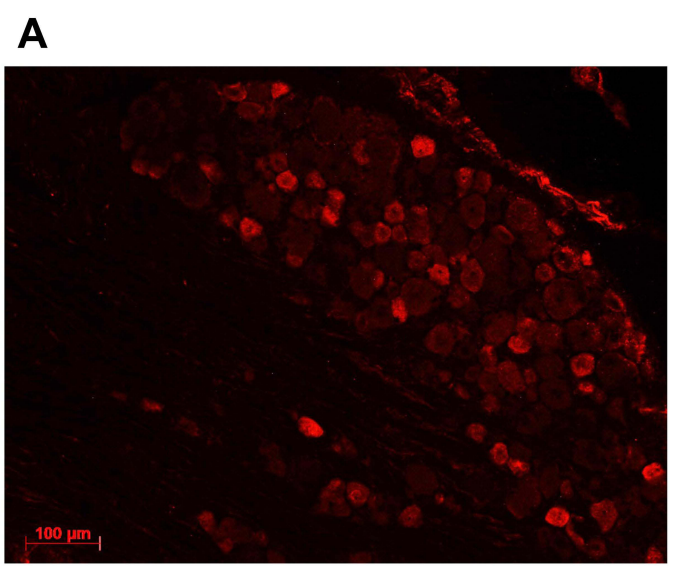

\section{B}

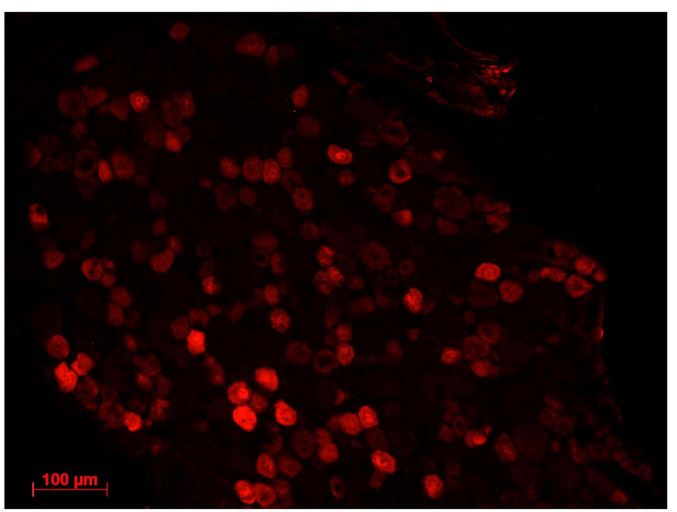

C
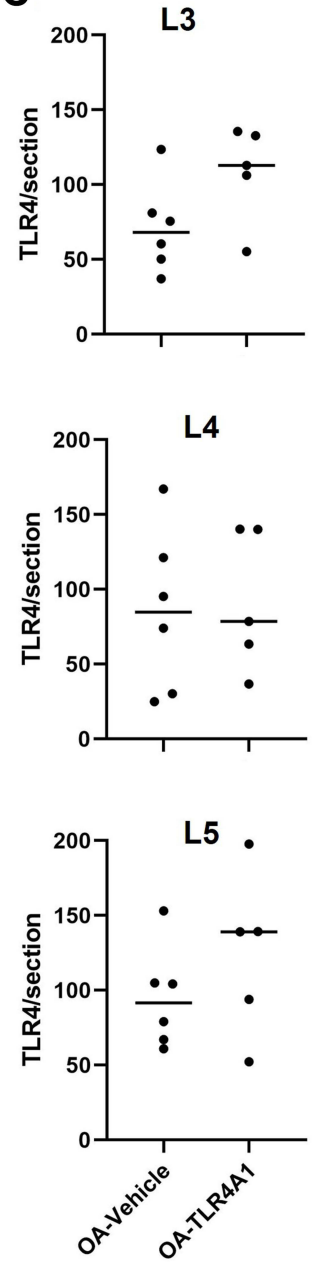

D

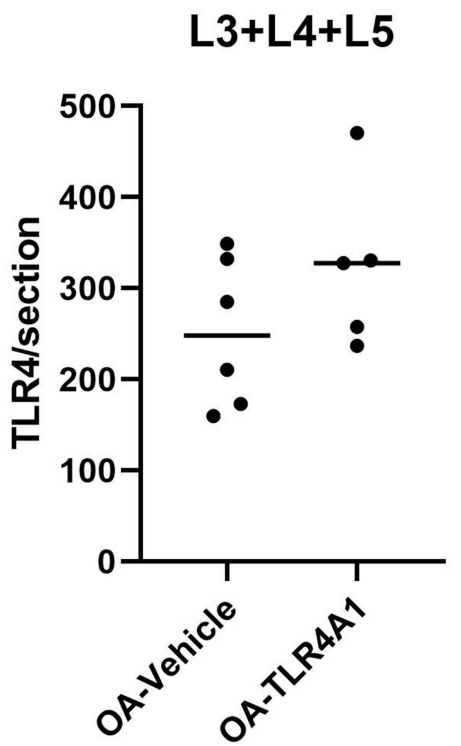

Figure 4 TLR4 expression in DRG neurons. A, (B) Representative images of immunofluorescence labelling for TLR4 in ipsilateral DRGs of OA-Vehicle (A) and OA-TLR4AI (B) animals. Immunofluorescence cell counting analysis for TLR4 cytoplasmic labelling in L3, L4 and L5 ipsilateral DRG of OA-Vehicle and OA-TLR4AI animals (C and D), after 15 days of chronic TLR4-AI administration.

TLR4 blockade, might account for the decrease in \%TIPPI in the second week of treatment.

As already mentioned, injured tissues in OA can generate several DAMPs that activate TLR4 present on the cartilage, bone and synovium, which will lead to a sustained production of several mediators with proinflammatory and pronociceptive effects. ${ }^{19}$ By mimicking OA, MIA injection might cause chondrocyte apoptosis and fibrosis at the articular joint, generating DAMPs. Being the TLR4 responsible for their recognition and for the expression of costimulatory molecules on antigen presenting cells, ${ }^{42,43}$ TLR4-A1 could be blocking this immune-mediated response, possibly by preventing the activation of the nuclear factor kappa-light-chain-enhancer of activated $\mathrm{B}$ cells (NF-kB) signaling pathway and the production of TNF- $\alpha$ and IL- $1 \beta$ at the joint, which will lead to a reduction in the excitability of primary afferent neurons and to a reduction of movement-related nociception. ${ }^{44}$ Park et $\mathrm{al}^{11}$ have also recently showed that intra-articular administration of TAP2, a peptide antagonist of TLR4, suppressed the expression of pro-inflammatory cytokines at the joint of OA animals and also reduced the secondary mechanical allodynia. ATF-3, an adaptive response gene induced by a variety of stress signals, ${ }^{45}$ is not expressed in most healthy intact neurons, but its expression is induced following nerve injury, ${ }^{46}$ being associated with neuroprotective and regenerative effects in the peripheral nervous system. ${ }^{47,48}$ ATF-3 can be induced by TLR4 signaling and behaves as a negative regulatory transcription factor in TLR pathways, controlling the inflammatory response, at least in antigen presenting cells (APCs), ${ }^{30}$ most likely through attenuation of NF-kB 


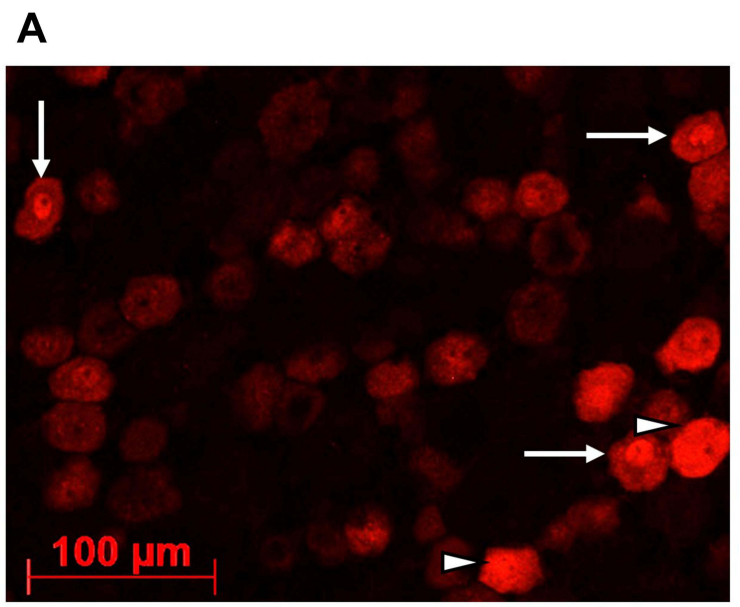

B
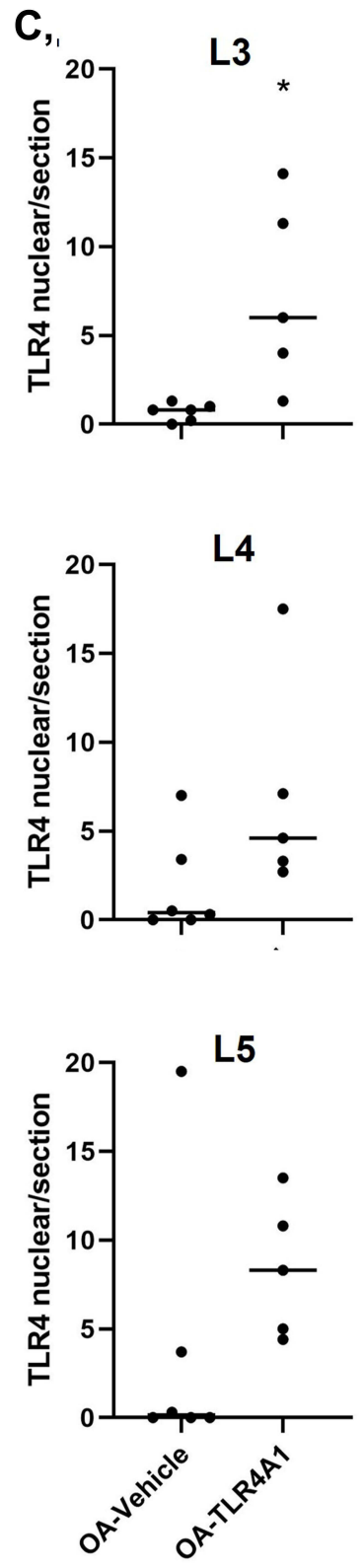

Figure 5 TLR4 nuclear expression in DRG neurons. (A) Representative image of TLR4 nuclear labelling (arrows) in the ipsilateral DRG of an OA-TLR4AI animal (amplification of Figure 4B). Immunofluorescence cell counting analysis for TLR4 nuclear labelling the L3, L4 and L5 ipsilateral DRG of OA-Vehicle and OA-TLR4AI animals (B and $\mathbf{C}$ ), after 15 days of chronic TLR4-AI administration. $* \mathrm{P}<0.05$ significantly different from OA-vehicle (Unpaired $t$-test).

activity reduction via direct interaction with the p65 subunit. ${ }^{49}$ Neuroinflammatory environments at the joints might be neurotoxic and trigger ATF-3 expression indirectly, most possibly through nerve damage. So, as the TLR4 pathway was being silenced by the TLR4 antagonist, it is possible that the inflammatory response was being suppressed, since reduction of pro-inflammatory cytokines have already been observed following administration of TLR4-A1, ${ }^{50}$ thus reducing the neurotoxic environment, leading to a decreased expression of ATF-3 in DRG neurons.

Since TLR4 has been identified in neurons within the $\mathrm{DRG},{ }^{51}$ it is possible that the primary afferent neurons directly detect PAMPs and DAMPs independently of classic immunocompetent cells, to send warning signals to the brain. ${ }^{44}$ Moreover, it has been shown that TLR4 expression in the DRG increases in neuropathic conditions. ${ }^{52}$ Thus, in this study, we also evaluated 


\section{A}

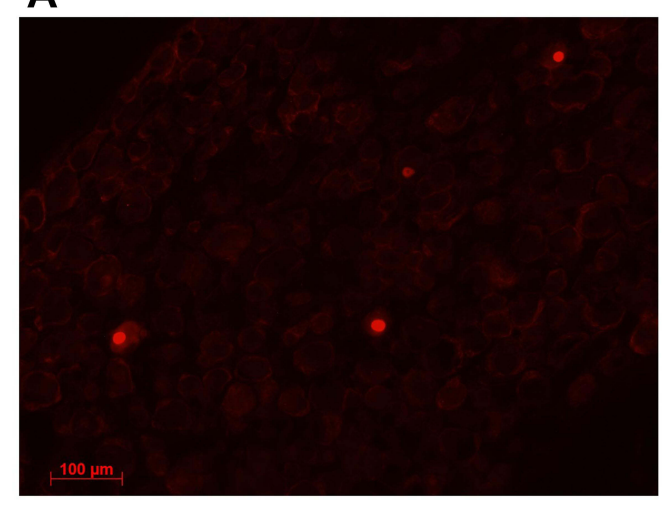

B

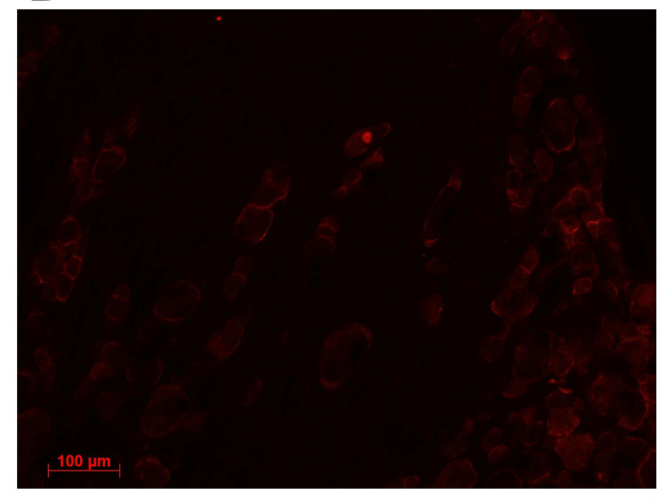

C
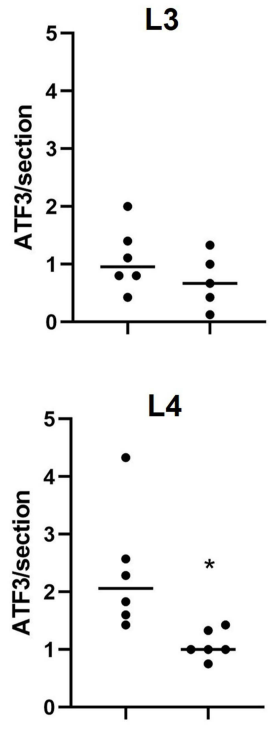

L5

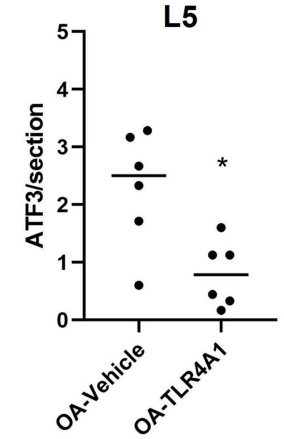

D

\section{L3+L4+L5}

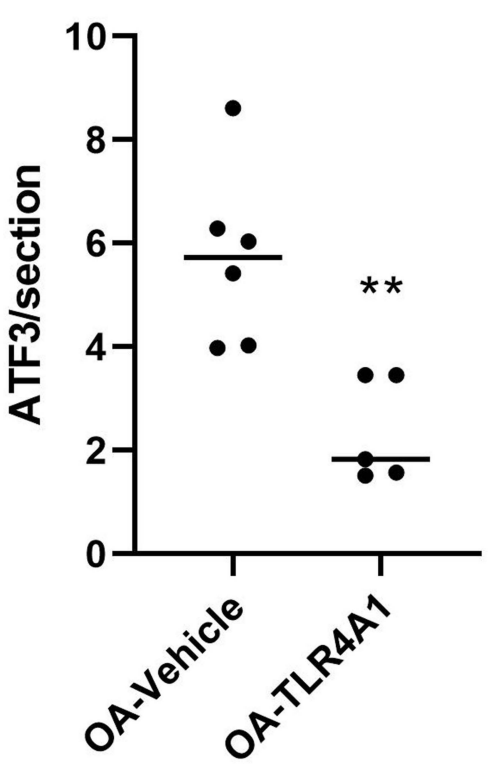

Figure 6 ATF-3 expression in DRG neurons. (A and B) Representative images of immunofluorescence labelling for ATF-3 in ipsilateral DRGs of OA-Vehicle (A) and OATLR4AI (B) animals. Immunofluorescence cell counting analysis for ATF-3 labelling (nuclear) in L3, L4 and L5 ipsilateral DRG of OA-Vehicle and OA-TLR4AI animals (C and D), after 15 days of chronic TLR4-AI administration. $* \mathrm{P}<0.05$, $* * \mathrm{P}<0.01$ significantly different from OA-vehicle (Unpaired $t$-test).

TLR4 expression in DRG after TLR4-A1 administration to assess if the antagonist was having an effect not only through its action on the joint but also at the DRG level. TLR4 administration did not decrease TLR4 expression in DRG neurons, which lead us to hypothesize that the antagonist has not a direct effect on the TLR4 expressed in the neuronal perikarya. In fact, from a pharmacodynamic perspective, given the low expected permeability of the TLR4A1 through the blood-brain barrier (it is a quaternary ammonium compound containing long alkyl chains), the effects observed could be preferentially mediated by peripheral blockage. However, DRG lack a protective surrounding capsular membrane, like the perineurium, and an efficient neurovascular barrier, like in the CNS, which indicates that, theoretically, DRG neurons can readily be targeted by systemic delivery approaches, such as intravenous injection. ${ }^{53}$ Furthermore, given the well-known presence of TLR4 receptors in primary sensory neurons ${ }^{54}$ as well as in resident and recruited macrophages, ${ }^{55}$ we cannot exclude an effect of TLR4-A1 at this level.

While the neuronal TLR4 expression was predominantly cytoplasmic, nuclear expression was also observed in the DRG of OA animals in our study. In addition, TLR4-A1 increased the neuronal nuclear expression of TLR4 in the L3 DRG, although the reason for this is unclear. Though this is not a common observation, other authors have already described nuclear expression of TLR4 in other cell types. ${ }^{56-58}$ However, the precise implication of this is not understood. Janardhan et $\mathrm{al}^{56}$ showed nuclear localization of TLR4 in lung cells, which colocalized with LPS. Yet, it was not clear if the TLR4-LPS complex was formed in the cell surface or in the cytoplasm prior to its migration into the nucleus, or whether this complex was formed in the nucleus with pre-existing TLR4. ${ }^{56}$ 
Recently, a protective role of TLR4 in cartilage homeostasis has been suggested, which might indicate a double-edge function of this receptor in OA pathophysiology. Chondrocytes isolated from TLR2 and TLR4 double knockout showed reduced anti-oxidative stress capacity and impaired autophagy flux, which could indicate chondrocyte dysfunction and compromise their homeostasis. ${ }^{59}$ In agreement, we observed no improvement in the histopathology of the joint of OA animals receiving the TLR4 antagonist, despite the reduction in nociception and in neuronal injury, which reinforces the possible distinct actions of the TLR4 pathway. This is in contrast with the study of Park et $\mathrm{al}^{11}$ which showed a reduction of cartilage degradation upon TAP2 administration. However, since inflammatory events involving TLR4 are complex, the two antagonists might produce different effects in the downstream pathway.

\section{Conclusions}

In conclusion, we hypothesize that DAMPs liberated by the damaged tissue in the extracellular joint milieu activate TLR4 present in different joint tissues, therefore triggering the TLR4 signalling cascade. As already described, TLR4 activation culminates in the transcription and expression of cytokines, chemokines, and other immune mediators that are neurotoxic to the primary afferent neurons innervating the joint. ATF-3 expression in DRG neurons is thus induced, as a negative feedback response, in an attempt to prevent additional neuronal injury. The TLR4 antagonist administration possibly interrupts this TLR4 cascade, decreasing the inflammatory environment, and as a consequence the ATF-3 expression, and improving the nociceptive symptoms.

\section{Ethics Approval}

All experimental procedures were approved by the Local Committee of Animal Welfare (ORBEA), with all adequate measures being taken to minimize pain or discomfort of the animals.

\section{Funding}

Granted by FEDER funds through COMPETE - Programa Operacional Factores de Competitividade (FCOMP-010124-FEDER-021359) and by National Funds through FCT - Fundação para a Ciência e a Tecnologia (PTDC/ SAU-NSC/119986/2010); and by Ministerio de Economia y Competitividad (SAF2012-40075-C02-02).

\section{Disclosure}

Ms Lígia Almeida reports grants and personal fees from Fundação para a Ciência e a Tecnologia (FCT), during the conduct of the study. The authors report no other potential conflicts of interest in this work.

\section{References}

1. Creamer P, Lethbridge-Cejku M, Hochberg MC. Where does it hurt? Pain localization in osteoarthritis of the knee. Osteoarthritis Cartilage. 1998;6(5):318-323. doi:10.1053/joca.1998.0130

2. McAlindon TE, Bannuru RR, Sullivan MC, et al. OARSI guidelines for the non-surgical management of knee osteoarthritis. Osteoarthritis Cartilage. 2014;22(3):363-388. doi:10.1016/j. joca.2014.01.003

3. Kloppenburg M, Kroon FP, Blanco FJ, et al. 2018 update of the EULAR recommendations for the management of hand osteoarthritis. Ann Rheum Dis. 2019;78(1):16-24. doi:10.1136/ annrheumdis-2018-213826

4. Simon LS. Nonsteroidal anti-inflammatory drugs and their risk: a story still in development. Arthritis Res Ther. 2013;15(Supp13): S1. doi:10.1186/ar4173

5. Chen K, Huang J, Gong W, Iribarren P, Dunlop NM, Wang JM. Tolllike receptors in inflammation, infection and cancer. Int Immunopharmacol. 2007;7(10):1271-1285. doi:10.1016/j. intimp.2007.05.016

6. Goldring SR, Scanzello CR. Plasma proteins take their toll on the joint in osteoarthritis. Arthritis Res Ther. 2012;14(2):111. doi:10.1186/ar3741

7. McCormack WJ, Parker AE, O'Neill LA. Toll-like receptors and NOD-like receptors in rheumatic diseases. Arthritis Res Ther. 2009;11(5):243. doi:10.1186/ar2729

8. Kalaitzoglou E, Griffin TM, Humphrey MB. Innate immune responses and osteoarthritis. Curr Rheumatol Rep. 2017;19(8):45. doi:10.1007/s11926-017-0672-6

9. Kim HA, Cho ML, Choi HY, et al. The catabolic pathway mediated by Toll-like receptors in human osteoarthritic chondrocytes. Arthritis Rheum. 2006;54(7):2152-2163. doi:10.1002/art.21951

10. Bobacz K, Sunk IG, Hofstaetter JG, et al. Toll-like receptors and chondrocytes: the lipopolysaccharide-induced decrease in cartilage matrix synthesis is dependent on the presence of toll-like receptor 4 and antagonized by bone morphogenetic protein 7. Arthritis Rheum. 2007;56(6):1880-1893. doi:10.1002/art.22637

11. Park H, Hong J, Yin Y, et al. TAP2, a peptide antagonist of toll-like receptor 4, attenuates pain and cartilage degradation in a monoiodoacetate-induced arthritis rat model. Sci Rep. 2020;10 (1):17451. doi:10.1038/s41598-020-74544-5

12. Campo GM, Avenoso A, Campo S, D'Ascola A, Nastasi G, Calatroni A. Small hyaluronan oligosaccharides induce inflammation by engaging both toll-like-4 and CD44 receptors in human chondrocytes. Biochem Pharmacol. 2010;80(4):480-490. doi:10.1016/j.bcp.2010.04.024

13. Okamura Y, Watari M, Jerud ES, et al. The extra domain A of fibronectin activates toll-like receptor 4. J Biol Chem. 2001;276 (13):10229-10233. doi:10.1074/jbc.M100099200

14. Sofat N, Robertson SD, Wait R. Fibronectin III 13-14 domains induce joint damage via Toll-like receptor 4 activation and synergize with interleukin-1 and tumour necrosis factor. $J$ Innate Immun. 2012;4(1):69-79. doi:10.1159/000329632

15. Schelbergen RF, Blom AB, van den Bosch MH, et al. Alarmins S100A8 and S100A9 elicit a catabolic effect in human osteoarthritic chondrocytes that is dependent on Toll-like receptor 4. Arthritis Rheum. 2012;64(5):1477-1487. doi:10.1002/art.33495 
16. Liu-Bryan R, Terkeltaub R. Chondrocyte innate immune myeloid differentiation factor 88-dependent signaling drives procatabolic effects of the endogenous Toll-like receptor 2/Toll-like receptor 4 ligands low molecular weight hyaluronan and high mobility group box chromosomal protein 1 in mice. Arthritis Rheum. 2010;62 (7):2004-2012. doi:10.1002/art.27475

17. Lepetsos P, Papavassiliou AG. ROS/oxidative stress signaling in osteoarthritis. Biochim Biophys Acta. 2016;1862(4):576-591. doi:10.1016/j.bbadis.2016.01.003

18. Canton J, Grinstein S. Priming and activation of NADPH oxidases in plants and animals. Trends Immunol. 2014;35(9):405-407. doi:10.1016/j.it.2014.07.007

19. Bruno K, Woller SA, Miller YI, et al. Targeting toll-like receptor-4 (TLR4)-an emerging therapeutic target for persistent pain states. Pain. 2018;159(10):1908-1915. doi:10.1097/j.pain.0000000 000001306

20. Tanga FY, Nutile-McMenemy N, DeLeo JA. The CNS role of Toll-like receptor 4 in innate neuroimmunity and painful neuropathy. Proc Natl Acad Sci U S A. 2005;102(16):5856-5861. doi:10.1073/pnas.0501634102

21. Wu FX, Bian JJ, Miao XR, et al. Intrathecal siRNA against toll-like receptor 4 reduces nociception in a rat model of neuropathic pain. Int J Med Sci. 2010;7(5):251-259. doi:10.7150/ijms.7.251

22. Lan LS, Ping YJ, Na WL, et al. Down-regulation of toll-like receptor 4 gene expression by short interfering RNA attenuates bone cancer pain in a rat model. Mol Pain. 2010;6:2. doi:10.1186/1744-8069-6-2

23. Creamer P, Hunt M, Dieppe P. Pain mechanisms in osteoarthritis of the knee: effect of intraarticular anesthetic. J Rheumatol. 1996;23 (6):1031-1036.

24. Moisio K, Eckstein F, Chmiel JS, et al. Denuded subchondral bone and knee pain in persons with knee osteoarthritis. Arthritis Rheum. 2009;60(12):3703-3710. doi:10.1002/art.25014

25. Ballegaard C, Riis RG, Bliddal $\mathrm{H}$, et al. Knee pain and inflammation in the infrapatellar fat pad estimated by conventional and dynamic contrast-enhanced magnetic resonance imaging in obese patients with osteoarthritis: a cross-sectional study. Osteoarthritis Cartilage. 2014;22(7):933-940. doi:10.1016/j.joca.2014.04.018

26. Hill CL, Hunter DJ, Niu J, et al. Synovitis detected on magnetic resonance imaging and its relation to pain and cartilage loss in knee osteoarthritis. Ann Rheum Dis. 2007;66(12):1599-1603. doi:10.1136/ $\operatorname{ard} .2006 .067470$

27. Barajon I, Serrao G, Arnaboldi F, et al. Toll-like receptors 3, 4, and 7 are expressed in the enteric nervous system and dorsal root ganglia. $J$ Histochem Cytochem. 2009;57(11):1013-1023. doi:10.1369/ jhc.2009.953539

28. Piazza M, Rossini C, Della Fiorentina S, et al. Glycolipids and benzylammonium lipids as novel antisepsis agents: synthesis and biological characterization. J Med Chem. 2009;52(4):1209-1213. doi: $10.1021 / \mathrm{jm} 801333 \mathrm{~m}$

29. Gilchrist M, Thorsson V, Li B, et al. Systems biology approaches identify ATF3 as a negative regulator of Toll-like receptor 4. Nature. 2006;441(7090):173-178. doi:10.1038/nature04768

30. Whitmore MM, Iparraguirre A, Kubelka L, Weninger W, Hai T, Williams BR. Negative regulation of TLR-signaling pathways by activating transcription factor-3. J Immunol. 2007;179 (6):3622-3630. doi:10.4049/jimmunol.179.6.3622

31. Ferreira-Gomes J, Adães S, Sousa RM, Mendonça M, CastroLopes JM. Dose-dependent expression of neuronal injury markers during experimental osteoarthritis induced by monoiodoacetate in the rat. Mol Pain. 2012;8:50. doi:10.1186/1744-8069-8-50

32. Piazza M, Yu L, Teghanemt A, Gioannini T, Weiss J, Peri F. Evidence of a specific interaction between new synthetic antisepsis agents and CD14. Biochemistry. 2009;48(51):12337-12344. doi:10.1021/ bi901601b
33. Peri F, Granucci F, Costa B, Zanoni I, Marinzi C, Nicotra F. Inhibition of lipid a stimulated activation of human dendritic cells and macrophages by amino and hydroxylamino monosaccharides. Angew Chem Int Ed Engl. 2007;46(18):3308-3312. doi:10.1002/ anie. 200604932

34. Peri F, Piazza M. Therapeutic targeting of innate immunity with toll-like receptor 4 (TLR4) antagonists. Biotechnol Adv. 2012;30 (1):251-260. doi:10.1016/j.biotechadv.2011.05.014

35. Zimmermann M. Ethical guidelines for investigations of experimental pain in conscious animals. Pain. 1983;16(2):109-110. doi:10.1016/0304-3959(83)90201-4

36. Ferreira-Gomes J, Adães S, Castro-Lopes JM. Assessment of movement-evoked pain in osteoarthritis by the knee-bend and CatWalk tests: a clinically relevant study. $J$ Pain. 2008;9 (10):945-954. doi:10.1016/j.jpain.2008.05.012

37. Neogi TSJ. Neurobiological mechanisms of osteoarthritic pain and analgesic treatment. In: Felson DTS, editor. Pain in Osteoarthritis. Wiley-Blackwell; 2009:233-254.

38. Grubb. Activation of sensory neurons in the arthritic joint. Felson DSH, In: editor. Pain in Osteoarthritis. Wiley-Blackwell; 2009: 25-38

39. French HP, Smart KM, Doyle F. Prevalence of neuropathic pain in knee or hip osteoarthritis: a systematic review and meta-analysis. Semin Arthritis Rheum. 2017;47(1):1-8. doi:10.1016/j. semarthrit.2017.02.008

40. Lu D, Chen J, Hai T. The regulation of ATF3 gene expression by mitogen-activated protein kinases. Biochem J. 2007;401(2):559-567. doi:10.1042/bj20061081

41. Jahn K, Deutschländer A, Stephan T, et al. Supraspinal locomotor control in quadrupeds and humans. Prog Brain Res. 2008;171:353-362. doi:10.1016/s0079-6123(08)00652-3

42. Abdollahi-Roodsaz S, Joosten LA, Roelofs MF, et al. Inhibition of toll-like receptor 4 breaks the inflammatory loop in autoimmune destructive arthritis. Arthritis Rheum. 2007;56(9):2957-2967. doi: $10.1002 /$ art. 22848

43. Lefebvre JS, Lévesque T, Picard S, et al. Extra domain A of fibronectin primes leukotriene biosynthesis and stimulates neutrophil migration through activation of toll-like receptor 4. Arthritis Rheum. 2011;63(6):1527-1533. doi:10.1002/art.30308

44. Liu T, Gao YJ, Ji RR. Emerging role of toll-like receptors in the control of pain and itch. Neurosci Bull. 2012;28(2):131-144. doi:10.1007/s12264-012-1219-5

45. Thompson MR, Xu D, Williams BR. ATF3 transcription factor and its emerging roles in immunity and cancer. $J$ Mol Med (Berl). 2009;87(11):1053-1060. doi:10.1007/s00109-009-0520-x

46. Tsujino H, Kondo E, Fukuoka T, et al. Activating transcription factor 3 (ATF3) induction by axotomy in sensory and motoneurons: a novel neuronal marker of nerve injury. Mol Cell Neurosci. 2000;15 (2):170-182. doi:10.1006/mcne.1999.0814

47. Gey M, Wanner R, Schilling C, Pedro MT, Sinske D, Knöll B. Atf3 mutant mice show reduced axon regeneration and impaired regeneration-associated gene induction after peripheral nerve injury. Open Biol. 2016;6(8):160091. doi:10.1098/rsob.160091

48. Seijffers R, Allchorne AJ, Woolf CJ. The transcription factor ATF-3 promotes neurite outgrowth. Mol Cell Neurosci. 2006;32(12):143-154. doi:10.1016/j.mcn.2006.03.005

49. Kwon JW, Kwon HK, Shin HJ, Choi YM, Anwar MA, Choi S. Activating transcription factor 3 represses inflammatory responses by binding to the p65 subunit of NF-кB. Sci Rep. 2015;5(1):14470. doi: $10.1038 /$ srep 14470

50. Palmer C, Peri F, Neumann F, Ahmad F, Leake DS, Pirianov G. The synthetic glycolipid-based TLR4 antagonist FP7 negatively regulates in vitro and in vivo haematopoietic and non-haematopoietic vascular TLR4 signalling. Innate Immun. 2018;24(7):411-421. doi:10.1177/ 1753425918798904 
51. Due MR, Piekarz AD, Wilson N, et al. Neuroexcitatory effects of morphine-3-glucuronide are dependent on Toll-like receptor 4 signaling. J Neuroinflammation. 2012;9(1):200. doi:10.1186/17422094-9-200

52. Jurga AM, Rojewska E, Makuch W, Mika J. Lipopolysaccharide from Rhodobacter sphaeroides (TLR4 antagonist) attenuates hypersensitivity and modulates nociceptive factors. Pharm Biol. 2018;56 (1):275-286. doi:10.1080/13880209.2018.1457061

53. Abram SE, Yi J, Fuchs A, Hogan QH. Permeability of injured and intact peripheral nerves and dorsal root ganglia. Anesthesiology. 2006;105(1):146-153. doi:10.1097/00000542-200607000-00024

54. Wadachi R, Hargreaves KM. Trigeminal nociceptors express TLR-4 and CD14: a mechanism for pain due to infection. $J$ Dent Res. 2006;85(1):49-53. doi:10.1177/154405910608500108

55. Olson JK, Miller SD. Microglia initiate central nervous system innate and adaptive immune responses through multiple TLRs. J Immunol. 2004;173(6):3916-3924. doi:10.4049/jimmunol.173.6.3916
56. Janardhan KS, McIsaac M, Fowlie J, et al. Toll like receptor-4 expression in lipopolysaccharide induced lung inflammation. Histol Histopathol. 2006;21(7):687-696. doi:10.14670/hh-21.687

57. Huhta H, Helminen O, Lehenkari PP, Saarnio J, Karttunen TJ, Kauppila JH. Toll-like receptors 1, 2, 4 and 6 in esophageal epithelium, Barrett's esophagus, dysplasia and adenocarcinoma. Oncotarget. 2016;7(17):23658-23667. doi:10.18632/oncotarget.8151

58. Wong Y, Sethu C, Louafi F, Hossain P. Lipopolysaccharide regulation of toll-like receptor-4 and matrix metalloprotease-9 in human primary corneal fibroblasts. Invest Ophthalmol Vis Sci. 2011;52 (5):2796-2803. doi:10.1167/iovs.10-5459

59. Wang Y, Zhao X, Liu-Bryan R. Role of TLR2 and TLR4 in regulation of articular chondrocyte homeostasis. Osteoarthritis Cartilage. 2020;28(5):669-674. doi:10.1016/j.joca.2020.01.011

\section{Publish your work in this journal}

The Journal of Pain Research is an international, peer reviewed, open access, online journal that welcomes laboratory and clinical findings in the fields of pain research and the prevention and management of pain. Original research, reviews, symposium reports, hypothesis formation and commentaries are all considered for publication. The manuscript management system is completely online and includes a very quick and fair peer-review system, which is all easy to use. Visit http:// www.dovepress.com/testimonials.php to read real quotes from published authors. 